\title{
Retrospective Comparative Study of the Effects of Dendritic Cell Vaccine and Cytokine-Induced Killer Cell Immunotherapy with that of Chemotherapy Alone and in Combination for Colorectal Cancer
}

\author{
Jingxiu Niu, ${ }^{1}$ Yanjie Ren, ${ }^{2}$ Tianyu Zhang, ${ }^{2}$ Xuejing Yang, ${ }^{2}$ Wei Zhu, ${ }^{1}$ Hui Zhu, \\ Jing Li, ${ }^{1}$ Jiali Li, ${ }^{1}$ and Yan Pang ${ }^{1}$ \\ ${ }^{1}$ Department of Oncology, Tianjin Union Medicine Center, No. 190 Jieyuan Road, Hongqiao District, Tianjin 300121, China \\ ${ }^{2}$ Shanghai Claison Biotechnology Co. Ltd., Shanghai, China \\ Correspondence should be addressed to Yan Pang; pangyancn@126.com
}

Received 8 May 2014; Revised 20 July 2014; Accepted 5 August 2014; Published 18 August 2014

Academic Editor: Shiwu Zhang

Copyright (C) 2014 Jingxiu Niu et al. This is an open access article distributed under the Creative Commons Attribution License, which permits unrestricted use, distribution, and reproduction in any medium, provided the original work is properly cited.

\begin{abstract}
Purpose. This retrospective study determined the delayed-type hypersensitivity (DTH) skin test and safety of dendritic cell (DC) vaccine and cytokine-induced killer (CIK) cell immunotherapy and the survival compared to chemotherapy in 239 colorectal cancer (CRC) patients. Methods. DTH and safety of the immunotherapy were recorded. The overall survival (OS) and disease free survival curves were compared according to the immunotherapy and/or chemotherapy received with Kaplan-Meier estimates. Results. Of the 70 patients who received immunotherapy, $62.86 \%$ had a positive DTH skin test, $38.57 \%$ developed fever, $47.14 \%$ developed insomnia, 38.57\% developed anorexia, 4.29\% developed joint soreness, and 11.43\% developed skin rash. For 204 resectable CRC patients, median survival time (MST) (198.00 days) was significantly longer in patients with immunotherapy plus chemotherapy than with chemotherapy alone $(106.00$ days $)(P=0.02)$. For 35 patients with unresectable or postsurgery relapsed CRC and who were confirmed to be dead, no statistical difference was observed in the MST between the patients treated with immunotherapy and with chemotherapy $(P=0.41)$. MST in the patients treated with chemotherapy plus immunotherapy was 154 days longer than that of patients treated with chemotherapy alone $(P=0.41)$. Conclusions. DC vaccination and CIK immunotherapy did not cause severe adverse effects, induce immune response against CRC, and prolong OS.
\end{abstract}

\section{Introduction}

Oxaliplatin and 5-FU-based chemotherapy are the standard treatment modalities for high-risk stage II, stage III, and stage IV CRC patients [1, 2]. Regimens of capecitabine, CapeOX, and FOLFOX 4 are also commonly used in clinical treatment, and they result in survival benefits. Oxaliplatin and/or 5-FU can kill both rapidly dividing CRC cells and normal cells $[3,4]$. As a result, hair loss, diarrhea, nausea, and vomiting are commonly observed in CRC patients undergoing chemotherapy treatment. In addition to these adverse effects, severe adverse events including myelosuppression, immunosuppression, and permanent organ damage to the heart, lung, liver, and kidneys can occur during chemotherapy in CRC patients [5]. Because of their poor general condition, advanced CRC patients often cannot withstand the toxic effects of chemotherapy and, therefore, do not receive adequate therapy. In addition to its toxicity, chemotherapy sensitivity declines over time $[6,7]$. CRC that recurs after an initial response to oxaliplatin and 5-FU tends to be resistant to subsequent chemotherapy with different drugs. Complete tumor eradication is rarely achieved in most advanced CRC patients treated with chemotherapy [7]. Because of the limited clinical benefit and toxicity of chemotherapy, immunotherapy may be a better option for improving OS in advanced CRC. Immunotherapy uses the body's immune system to attack cancer cells. Antigen-specific $\mathrm{T}$ cell dysfunction is common in cancer patients. As a result, tumor cells escape immune surveillance. Restoring the immune system may be a viable option to improve 
cancer treatment. Immunotherapy may be a promising and safe approach for cancer eradication $[8,9]$. Unlike routine therapies, immunotherapy may induce an effective antitumor immune response without adverse effects. Immunotherapy with dendritic cell (DC) and cytokine-induced killer (CIK) cells is targeted to kill residual cancer cells, which are the main cause of cancer recurrence and metastasis. DC and CIK immunotherapy has been shown to be well tolerated with excellent compliance in cancer patients $[10,11]$.

The purpose of this study was to compare the therapeutic efficacy in terms of survival prolongation of DC cells and CIK cells immunotherapy and chemotherapy alone and in combination in advanced CRC patients.

\section{Patients and Methods}

2.1. Patients. CRC patients who were treated at the Department of Oncology, Tianjin Union Medicine Center, from February 1, 2012 to September 30, 2013 were included in the study. Inclusion criteria consist of: (1) patients with histologically or cytologically diagnosed CRC and adequate kidney liver, coagulation, and bone marrow function; (2) resectable (stage II and stage III) CRC patients who accepted primary tumor resection and postoperative adjuvant chemotherapy and had an elevated serum carcinoembryonic antigen (CEA) level before surgery and a normal serum CEA level $(5 \mathrm{ng} / \mathrm{mL})$ within 1 month after surgery; and (3) advanced CRC patients (relapsed or metastatic CRC after surgery and unresectable CRC) who were confirmed to be dead of any cause.

The resectable CRC patients were divided into 2 groups according to the treatment they received. Patients who received routine adjuvant postsurgery chemotherapy alone were defined as group $\mathrm{S}+\mathrm{C}$ (control group). Patients who received DC and CIK cells immunotherapy plus chemotherapy within 6 months after surgery were defined as group $S$ $+\mathrm{C}+\mathrm{I}$ (immunotherapy group). Advanced CRC patients were divided into 3 groups according to the treatment modalities they received: group I, DC cells and CIK cells immunotherapy; group C, chemotherapy; and group I + C, DC vaccine and CIK cell immunotherapy plus chemotherapy. The interval between immunotherapy and chemotherapy had to be less than 3 months; it is to ensure that the result of clinical efficacy is the result of immunotherapy and chemotherapy function together rather than unilateral.

2.2. Design of DC Cells and CIK Cells Therapy. The schedule of DC and CIK therapy was performed in accordance with the "Treatment with Autologous Immune Cells (T cells, NK cells)" class III medical techniques policy of the Ministry of Health of China. This study protocol was approved by ethical committees of the hospital. Written informed consent was obtained from each patient before treatment initiation. Adequate renal and coagulation function and peripheral lymphocyte and monocyte numbers greater than $1 \times 10^{9} / \mathrm{L}$ were required for patients to receive DC cells and CIK cells therapy.

2.3. Collection of Peripheral Blood Mononuclear Cells. On day 0, peripheral blood mononuclear cells (PBMCs) were collected by leukapheresis using a Fresenius KABI System (Germany) with an electrocardiogram monitoring system. PBMCs were cultured overnight and adherent cells (monocytes) and nonadherent cells (lymphocytes) were separated.

2.4. Preparation of DCs and CIK Cells. Tumor lysate was first prepared for pulsing DCs. A single cell suspension of SW480 colon cancer cell line was dissociated by ultrasound and centrifugation at $600 \times \mathrm{g}$ for $30 \mathrm{~min}$, and the supernatants were collected as tumor lysate. DCs were obtained by culturing the adherent cells through stimulating with tumor lysate, granulocyte macrophage colony-stimulating factor, interleukin (IL)-4, and tumor necrosis factor for 7 days [11, 12]. For preparation of CIK cells, SW480 cells CIK cells were obtained by culturing the nonadherent cells through stimulating with interferon $\gamma, \mathrm{CD} 3$ monoclonal antibody, and IL-2 for 10 days.

2.5. Quality Control of DCs and CIK Cells. The immune phenotypes of HLA2DR, CD80, and CD83 for DCs and CD3, CD8, and CD56 for CIK were analyzed by flow cytometry [13]. The bacteria, fungus, and endotoxin levels in cultured DC and CIK samples met the release criteria for infusion $[10,14,15]$.

2.6. Schedule of DC Cells and CIK Cells Infusion. DCs $(1 \times$ $10^{7}$ ) in $100 \mathrm{~mL} 0.9 \%$ normal saline (NS) were intravenously infused on days 8,15 , and 22 and injected intradermally on days 29,36 , and 43 . CIK cells $\left(1 \times 10^{9}\right)$ in $100 \mathrm{~mL} \mathrm{NS}$ were intravenously infused on days $11,12,13$, and 14. An interval of 4-6 hours was needed to receive immunotherapy and chemotherapy within the same day [15].

2.7. Delayed-Type Hypersensitivity Testing of DC Vaccine and CIK Cell Infusion. For delayed-type hypersensitivity (DTH) tests, $4 \mu \mathrm{g}$ of tumor lysate was intradermally injected 1 week after the last DC infusion and results were examined 48 hours later. An induration greater than $2 \mathrm{~mm}$ in diameter around the injection site was considered as a positive DTH response (Table 2).

2.8. Safety Evaluation of DC Cells and CIK Cells Immunotherapy. Adverse effects such as fever, insomnia, anorexia, joint soreness, and skin rash were evaluated during DC cells and CIK cells infusion. Several adverse effects occurred simultaneously in the same patient (Table 2).

2.9. Chemotherapy Schedules. Capecitabine was administered orally twice daily at a dose of $1000 \mathrm{mg} / \mathrm{m}^{2}$ on day 1 to day 14 . This regimen was repeated every 3 weeks [16, 17]. For the capecitabine plus oxaliplatin (CapeOX) regimen, oxaliplatin was intravenously infused at a dose of $130 \mathrm{mg} / \mathrm{m}^{2}$ on day 1 , and capecitabine was administered orally twice daily at a dose of $1000 \mathrm{mg} / \mathrm{m}^{2}$ on day 1 to day 14 . The regimen was repeated every 3 weeks. The FOLFOX 4 (oxaliplatin, leucovorin [LV], and 5-FU) regimen consisted of intravenous oxaliplatin infusion $\left(85 \mathrm{mg} / \mathrm{m}^{2}\right)$ on day 1 , intravenous $\mathrm{LV}$ 
infusion $\left(200 \mathrm{mg} / \mathrm{m}^{2}\right)$ on day 1 and day 2 , and intravenous bolus injection $\left(400 \mathrm{mg} / \mathrm{m}^{2}\right)$ and $22 \mathrm{~h}$ infusion $\left(600 \mathrm{mg} / \mathrm{m}^{2}\right)$ of 5-FU on day 1 and day 2 . This regimen was repeated every 2 weeks. To receive chemotherapy, patients were required to have adequate kidney, liver, and bone marrow function [1720].

2.10. Follow-Up. Overall survival (OS) was recorded for the advanced CRC patients. For the resectable CRC patients, CEA levels were measured before operation and within 1 month after operation, and patients were followed up until November 14, 2013. The follow-up occurred 3 to 4 weeks postoperatively. Serum CEA levels were monitored every 3 weeks just before receiving adjuvant chemotherapy. Serum CEA levels were recorded every 3 months for the first 2 years of follow-up and thereafter every 6 months. Based on the restrictions presented below, the time from the date of surgery to the date of the first rise in serum CEA level above the normal upper limit reflected the duration of tumor-free status in patients, and, therefore, it was used to provide an estimate of disease-free survival (DFS). Patients with serum CEA levels more than $5 \mathrm{ng} / \mathrm{mL}$ before surgery, which was the reference cutoff in our hospital, were considered to have CEAproducing tumors, and, therefore, CEA could be used as an index for evaluation of tumor recurrence. Patients with serum CEA levels below $5 \mathrm{ng} / \mathrm{mL}$ within 1 month of surgery were considered as a radical surgery. Patients whose serum CEA level was above the normal upper limit 3 consecutive times during follow-up were considered to have tumor recurrence [21-24].

2.11. Data Collection and Statistical Analysis. The patients were followed up until November 14, 2013. In advanced CRC patients, OS was defined as the time from the date of study enrollment to the date of death from any cause. In resectable CRC patients, DFS was defined as the time from the date of the first rise in serum CEA level above the normal upper limit to the date of surgery.

Clinical data of the patients including diagnostic procedures and treatment were collected from the inpatients electronic medical records of our hospital and reanalyzed using EpiData database (version 3.02). Particular attention was paid to collecting data related to primary rumor resection, chemotherapy, and DC cells and CIK cells immunotherapy. Statistical analyses were carried out using the SPSS (version 19.0) statistical software package, which was docked with the EpiData database. DFS and OS curves were calculated using the Kaplan-Meier method. A $P$ value less than 0.05 was considered statistically significant.

\section{Results}

3.1. Patient Characteristics. A total of 239 CRC patients (204 resectable CRC patients and 35 advanced CRC patients) were enrolled in the study during an 8-month period from February 1, 2012 to September 30, 2012 and followed up until November 14, 2013. Patient characteristics are shown in Table 1. Of the 239 patients, 133 male patients and 106
TABLE 1: Characteristics of patients.

\begin{tabular}{|c|c|c|c|}
\hline \multirow{2}{*}{ Characteristics } & \multicolumn{3}{|c|}{ Number } \\
\hline & Resectable & Advanced & Total \\
\hline Number & 204 & 35 & 239 \\
\hline \multicolumn{4}{|l|}{ Age (years) } \\
\hline Range & $28-83$ & $40-86$ & $28-86$ \\
\hline Mean \pm SD & $61.74 \pm 10.28$ & $62.1 \pm 8.10$ & $64.2 \pm 12.05$ \\
\hline \multicolumn{4}{|l|}{ Gender } \\
\hline Male & 116 & 17 & 133 \\
\hline Female & 88 & 18 & 106 \\
\hline \multicolumn{4}{|l|}{ Tumor location } \\
\hline Colon & 74 & 18 & 92 \\
\hline Rectum & 130 & 17 & 147 \\
\hline \multicolumn{4}{|l|}{ Differentiation degrees } \\
\hline High & 1 & 0 & 1 \\
\hline Middle & 161 & 27 & 188 \\
\hline Low & 42 & 8 & 50 \\
\hline \multicolumn{4}{|l|}{ UICC stages } \\
\hline I & 0 & 0 & 0 \\
\hline II & 85 & 0 & 85 \\
\hline III & 119 & 0 & 119 \\
\hline IV + recurrence & 0 & 35 & 35 \\
\hline \multicolumn{4}{|c|}{ Background of treatments } \\
\hline Surgery & 204 & 24 & 228 \\
\hline Radiology & 0 & 8 & 8 \\
\hline Chemotherapy & 204 & 28 & 232 \\
\hline DCs + CIK therapy & 74 & 24 & 98 \\
\hline
\end{tabular}

TABLE 2: Side effects in immunotherapy with DCs and CIK cells in immunotherapy group $(n=70)$.

\begin{tabular}{lcc}
\hline \multicolumn{2}{l}{ Characteristics Number of positives (\%) } & Number of negatives (\%) \\
\hline DTH & $44(62.86 \%)$ & $26(37.14 \%)$ \\
Fever & $27(38.57 \%)$ & $43(61.43 \%)$ \\
Insomnia & $33(47.14 \%)$ & $37(52.86 \%)$ \\
Anorexia & $27(38.57 \%)$ & $43(61.43 \%)$ \\
Joint soreness & $3(4.29 \%)$ & $67(95.71 \%)$ \\
Skin rash & $8(11.43 \%)$ & $62(88.57 \%)$ \\
\hline
\end{tabular}

female patients were with a mean age of $64.2 \pm 12.05$ (range 28-86) years. The primary tumor was located in the colon and rectum in 92 patients and 147 patients, respectively. One patient, 188 patients, and 50 patients had well-differentiated, moderately differentiated, and poorly differentiated tumors, respectively. Of the unresectable CRC patients, $24(20.8 \%)$ patients underwent primary tumor resection, $8(8.3 \%)$ patients received radiotherapy, 28 patients received chemotherapy, and 24 patients received DC cells and CIK cells immunotherapy. Of the 28 patients who received chemotherapy, 5 patients received the capecitabine regimen, 6 patients received the CapeOX regimen, 12 patients received the FOLFOX 4 regimen, and 5 patients received other regimens. 
TABLE 3: Serum CEA levels of presurgery, postsurgery, and tumor recurrence in both immunotherapy group and control group.

\begin{tabular}{lccc}
\hline Group & Total & Immunotherapy group & Control group \\
\hline Presurgery & & & $P$ value \\
$\quad$ Number & 204 & 74 & 130 \\
Mean \pm SD & $15.88 \pm 20.63$ & $13.79 \pm 11.99$ & $17.05 \pm 24.13$ \\
$\quad$ Range & $10-48$ & $10-48$ & $10-48$ \\
Postsurgery & & & 0.13 \\
Number & 204 & 74 & 130 \\
Mean \pm SD & $2.78 \pm 1.11$ & $2.91 \pm 1.10$ & $2.70 \pm 1.12$ \\
Range & $2-5$ & $2-5$ & $2-5$ \\
Tumor recurrence & $48 / 204$ & $17 / 74(23.0 \%)$ & $31 / 130(23.8 \%)$ \\
Number/total number & $8.21 \pm 5.96$ & $7.86 \pm 6.05$ & $8.40 \pm 6.00$ \\
Mean \pm SD & $5-10$ & $5-10$ & $5-10$ \\
Range & & 0.89 \\
\hline
\end{tabular}

Of the 204 resectable CRC patients, 85 patients had stage II disease and 119 patients had stage III disease. These 204 patients were divided into 2 groups according to the treatment they received. The control group $(n=130)$ received primary tumor resection followed by adjuvant chemotherapy. The immunotherapy group $(n=74)$ received primary tumor resection followed by adjuvant chemotherapy plus adjuvant DC cells and CIK cells immunotherapy. The 35 advanced CRC patients were divided into 3 groups according to the treatment they received: group $\mathrm{C}(n=11)$, chemotherapy alone; group I $(n=7)$, DC cells and CIK cells immunotherapy alone; and group I + C $(n=17)$, DC cells and CIK cells immunotherapy plus chemotherapy.

3.2. Immune Response and Safety of DC Cells and CIK Cells Therapy. Immune response and safety of DC cells and CIK cells therapy were recorded in 70 of the 74 patients in group I. These parameters were not evaluated in 4 patients because of incomplete follow-up data. Of the 70 group I patients, 44 $(62.86 \%)$ patients had a positive immune response based on the DTH skin test. Twenty-seven (38.57\%) patients developed fever, 33 (47.14\%) patients developed insomnia, 27 (38.57\%) patients developed anorexia, 3 (4.29\%) patients developed joint soreness, and $8(11.43 \%)$ patients developed skin rash (Table 2). Severe adverse events were not observed.

3.3. Serum CEA Levels in Resectable CRC Patients. Mean CEA level was significantly lower after operation compared with before operation in the 204 resectable CRC patients $(P=$ 0.81 ). Mean serum CEA level before and after surgery was $15.88 \pm 20.63$ (range 5-48) ng/mL and $2.78 \pm 1.11$ (range 548) $\mathrm{ng} / \mathrm{mL}$, respectively.

At the end of follow-up, serum CEA levels significantly rose in 48 of the 204 resectable CRC patients including 17 of the $74(23.0 \%)$ immunotherapy group patients and 31 of the $130(23.8 \%)$ control group patients $(P=0.58)$. The mean serum CEA level of these patients was $8.21 \pm 5.96$ (range $5-48) \mathrm{ng} / \mathrm{mL}$. Presurgery and postsurgery CEA levels and tumor recurrence were similar between the immunotherapy and control groups (Table 3).
TABLE 4: Comparison of MST in immunotherapy group and control group.

\begin{tabular}{lccccc}
\hline Group & Number & $\begin{array}{c}\text { MST } \\
(\text { days })\end{array}$ & $\begin{array}{c}\Delta \text { MST } \\
(\text { days })\end{array}$ & $\chi^{2}$ & $(P$ value $)$ \\
\hline $\begin{array}{l}\text { Immunotherapy } \\
\text { Control }\end{array}$ & 17 & 198.00 & 92.00 & 5.109 & 0.02 \\
\hline
\end{tabular}

3.4. DFS Based on Serum CEA Level. The 204 resectable CRC patients were followed up for $489.2 \pm 160.4$ (range 441652) days. At the end of follow-up, serum CEA levels were increased in 48 of the 204 patients including 17 patients in the immunotherapy group and 31 patients in the control group. Median survival time (MST) was significantly longer in the immunotherapy group than in the control group $(P=0.02)$ (Table 4$)$. MST was prolonged for 92 days in the immunotherapy group compared with the control group (198 days versus 106 days). The DFS curves for the immunotherapy and control groups are shown in Figure 1.

3.5. Comparison of OS and MST between Chemotherapy and Immunotherapy in Advanced CRC Patients. MSTs of the I, $\mathrm{C}$, and I $+\mathrm{C}$ groups are shown in Table 5. MST was not significantly different between the I and C groups (249 days versus 110 days; $P=0.41)$. MST was significantly longer in the $\mathrm{I}+\mathrm{C}$ group than in the $\mathrm{C}$ group $(P=0.04)$. MST was prolonged 154 days in the $\mathrm{I}+\mathrm{C}$ group compared with the C group (264 versus 110 days). MST was not significantly different between the I and I + C groups (249 days versus 264 days; $P=0.47$ ). The $\mathrm{OS}$ curves for the $\mathrm{I}, \mathrm{C}$, and $\mathrm{I}+\mathrm{C}$ groups are shown in Figure 2.

\section{Discussion}

DTH as an indicator of immune response can serve as an efficacy end point for DC cells and CIK cells immunotherapy. OS is a powerful index to measure therapeutic efficacy in advanced CRC. Indices to evaluate therapeutic efficacy in early-stage CRC postsurgery are lacking. CEA is the most widely used tumor marker for the management of CRC 


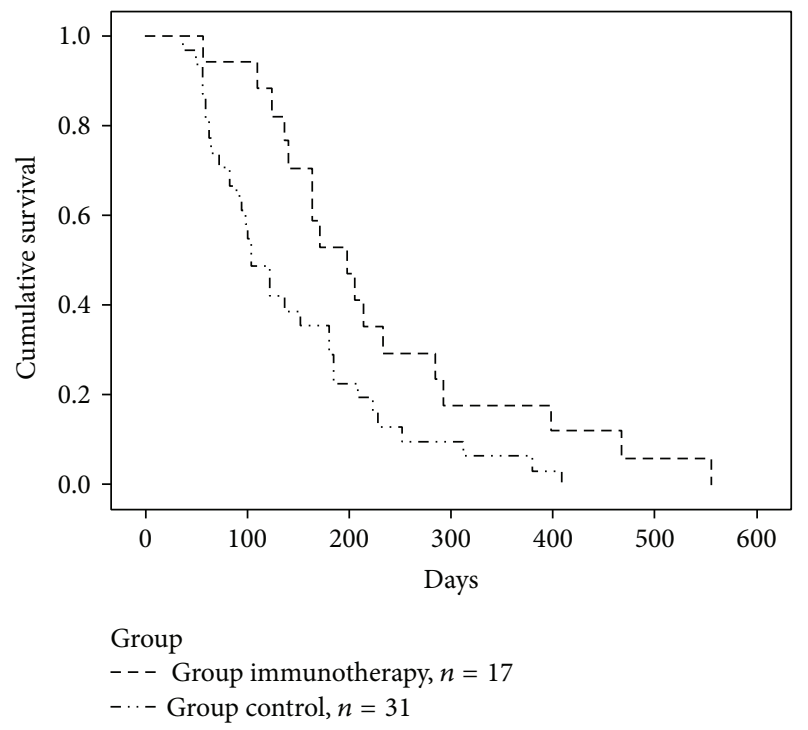

FIGURE 1: DFS curves by the Kaplan-Meier estimate in immunotherapy group and control group $(P=0.024)$.

TABLE 5: Comparison of MST for patients with chemotherapy, immunotherapy alone, and immunotherapy plus chemotherapy of DC vaccine and CIK cells.

\begin{tabular}{lccccc}
\hline Group & Number & MST (day) & $\Delta$ MST & $\chi^{2}$ & $(P$ value $)$ \\
\hline C versus I & & & & & \\
$\quad$ C & 11 & 110 & 139 & 0.694 & 0.41 \\
$\quad$ I & 7 & 249 & & & \\
C versus I + C & & & & & \\
$\quad$ C & 11 & 110 & 154 & 4.127 & 0.04 \\
$\quad$ I + C & 17 & 264 & & & \\
I versus I + C & & & & & \\
$\quad$ I & 7 & 249 & 15 & 0.535 & 0.47 \\
I + C & 17 & 264 & & & \\
\hline
\end{tabular}

$[25,26]$. CEA is currently used to detect recurrent disease after curative resection and is believed to be effective indicator of postoperative mortality. Postoperative CEA level is an important indicator for both OS and DFS rates in CRC patients. CEA is an early marker for tumor recurrence. CEA level can detect tumor recurrence approximately 5 months earlier compared with clinical symptoms and imaging diagnosis. Quantitative measurement of serum CEA can be readily, easily, and inexpensively obtained. In this study, patients with serum CEA levels below $5 \mathrm{ng} / \mathrm{mL}$ before surgery were excluded because they were considered to have non-CEA-producing tumors and, therefore, CEA could not be used as an index for evaluation of tumor recurrence. The high false positive rate of CEA testing in CRC, which often results from incidental rises in CEA caused by benign gastrointestinal disorders, has been a main problem with its use. Serum CEA remains in the circulation for a period of time and requires several weeks to return to normal. It is believed that incidental rises in CEA can be filtered, and a higher specificity is expected by repetitive measurements of

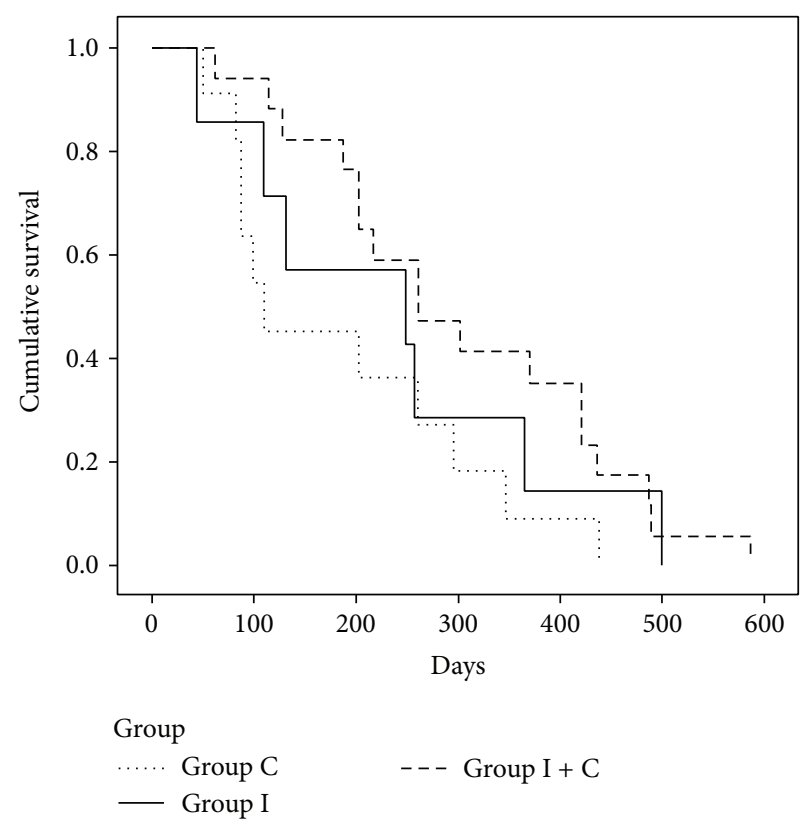

FIgURE 2: OS curves by the Kaplan-Meier estimate for patients with chemotherapy, immunotherapy alone, and immunotherapy plus chemotherapy of DC vaccine and CIK.

CEA at an interval of several weeks. In our study, a rise in the serum CEA level was defined as a CEA level above the normal upper limit for 3 consecutive follow-ups. Abnormal serum CEA levels in some patients persisted after surgery, which usually indicates the presence of residual microscopic disease. Patients with serum CEA levels above $5 \mathrm{ng} / \mathrm{mL}$ within 1 month after surgery were excluded from this study, because they were considered as nonradical surgery. If there is no residual disease after tumor resection, the serum CEA level should remain within normal range. If the CEA level rises, it is possible that a persistent source of CEA, such as a hidden metastasis or recurrent tumor is present. Based on these circumstances, the period from the date of surgery to that of the first rise in serum CEA level above the normal upper limit reflects the duration of tumor-free status, and this might provide another method to measure DFS [27, 28].

Based on the clinical observations of our study, 44 (62.86\%) patients developed a positive immune response to DC cells and CIK cells immunotherapy based on the DTH skin test. Immunotherapy-related adverse effects including fever, insomnia, anorexia, joint soreness, and skin rash were observed. In general, these adverse effects were mild and resolved without the need for additional treatment. Severe adverse events were not observed. A rise in serum CEA level was detected in 17 of the $74(23.0 \%)$ patients in the immunotherapy group and 31 of the $130(23.8 \%)$ patients in the control group. Although the number of patients with an elevated CEA level during follow-up was not significantly different between the 2 groups, MST, defined as the time from the date of surgery to the date of the first rise in serum CEA level, was significantly longer (92 days) in the immunotherapy group than that in the control group. MST 
was not significantly different between patients who received immunotherapy and those who received chemotherapy. MST was 154 days longer in the patients who received chemotherapy plus immunotherapy than in patients who received chemotherapy alone. This indicates that DC vaccine and CIK cell immunotherapy and chemotherapy may have a similar effect on survival in advanced CRC patients. Combined immunotherapy and chemotherapy may have a synergistic effect on survival compared with chemotherapy alone.

DC cells and CIK cells immunotherapy can induce an immune response against CRC and prolong OS and DFS. The therapy was safe and no severe adverse effects were observed. DC cells and CIK cells immunotherapy and chemotherapy had a similar survival benefit in CRC patients. Combined immunotherapy and chemotherapy had a synergistic effect on survival compared with chemotherapy alone. Immunotherapy represents a viable treatment option to benefit CRC patients.

\section{Conclusion}

DC vaccination and CIK cell therapy were safe and no severe adverse effects were observed. DC cells and CIK cells therapy were able to stimulate the patients to use their own immune system against cancer. As a result, DFS and OS were prolonged. Immunotherapy and chemotherapy had the same effect on survival in CRC patients. Combined immunotherapy and chemotherapy may have a synergistic effect on survival compared with chemotherapy alone [2931].

\section{Conflict of Interests}

The authors declare that there is no conflict of interests regarding the publication of this paper.

\section{Acknowledgment}

The present study was supported in part by the Tianjin Municipal Science and Technology Commission (no. 12ZCDZSY17100).

\section{References}

[1] S. Loi, S. Y. K. Ngan, R. J. Hicks et al., "Oxaliplatin combined with infusional 5-fluorouracil and concomitant radiotherapy in inoperable and metastatic rectal cancer: a phase I trial," British Journal of Cancer, vol. 92, no. 4, pp. 655-661, 2005.

[2] A. Gunnlaugsson, P. Nilsson, E. Kjellén, and A. Johnsson, “The effect on the small bowel of 5-FU and oxaliplatin in combination with radiation using a microcolony survival assay," Radiation Oncology, vol. 4, no. 1, article 61, 2009.

[3] S. Tsuji, Y. Midorikawa, T. Takahashi et al., "Potential responders to FOLFOX therapy for colorectal cancer by Random Forests analysis," British Journal of Cancer, vol. 106, no. 1, pp. 126-132, 2012.

[4] S. Satram-Hoang, L. Lee, S. Yu et al., "Comparative effectiveness of chemotherapy in elderly patients with metastatic colorectal cancer," Journal of Gastrointestinal Cancer, vol. 44, no. 1, pp. 7988, 2013.

[5] H. K. Sanoff, W. R. Carpenter, J. Freburger et al., "Comparison of adverse events during 5-fluorouracil versus 5-fluorouracil/ oxaliplatin adjuvant chemotherapy for stage III colon cancer: a population-based analysis," Cancer, vol. 118, no. 17, pp. 43094320, 2012.

[6] K. Salem, C. O. Brown, J. Schibler, and A. Goel, "Combination chemotherapy increases cytotoxicity of multiple myeloma cells by modification of nuclear factor (NF) $-\kappa \mathrm{B}$ activity," Experimental Hematology, vol. 41, no. 2, pp. 209-218, 2013.

[7] A. Dasgupta, J. E. Shields, and H. Trent Spencer, “Treatment of a solid tumor using engineered drug-resistant immunocompetent cells and cytotoxic chemotherapy," Human Gene Therapy, vol. 23, no. 7, pp. 711-721, 2012.

[8] B. A. Fox, D. J. Schendel, L. H. Butterfield et al., "Defining the critical hurdles in cancer immunotherapy," Journal of Translational Medicine, vol. 9, no. 1, article 214, 2011.

[9] A. Hoos, A. M. M. Eggermont, S. Janetzki et al., "Improved endpoints for cancer immunotherapy trials," Journal of the National Cancer Institute, vol. 102, no. 18, pp. 1388-1397, 2010.

[10] T. Yang, Y. Xiang, Y. Li, J. Shao, Q. Li, and H. Yu, "Clinical study of co-treatment with DC-CIK cells for advanced solid carcinomas," The Chinese-German Journal of Clinical Oncology, vol. 10, no. 6, pp. 354-359, 2011.

[11] R. Lubong-Sabado and N. Bhardwaj, "Directing dendritic cell immunotherapy towards successful cancer treatment," Immunotherapy, vol. 2, no. 1, pp. 37-56, 2010.

[12] N. Janikashvili, N. Laemonier, and E. Katsanis, "Personalized dendritic cell-based tumor immunotherapy," Immunotherapy, vol. 2, no. 1, pp. 57-68, 2010.

[13] X. Li, B. Xu, J. Wu et al., "Review of Chinese clinical trials on CIK cell treatment for malignancies," Clinical and Translational Oncology, vol. 14, no. 2, pp. 102-108, 2012.

[14] Y. Cui, X. Yang, W. Zhu, J. Li, X. Wu, and Y. Pang, "Immune response, clinical outcome and safety of dendritic cell vaccine in combination with cytokine-induced killer cell therapy in cancer patients," Oncology Letters, vol. 6, no. 2, pp. 537-541, 2013.

[15] Y. Liu, W. Zhang, B. Zhang, X. Yin, and Y. Pang, "DC vaccine therapy combined concurrently with oral capecitabine in metastatic colorectal cancer patients," Hepato-Gastroenterology, vol. 60 , no. 122, pp. 23-27, 2013.

[16] W. Ling, J. Fan, Y. Ma, and H. Wang, "Capecitabine-based chemotherapy for metastatic colorectal cancer," Journal of Cancer Research and Clinical Oncology, vol. 137, no. 6, pp. 927938, 2011.

[17] Y. Li, J. Li, M. Lu, X. Wang, and L. Shen, "Capecitabine maintenance therapy after first-line chemotherapy in patients with metastatic colorectal cancer," Chinese Journal of Cancer Research, vol. 22, no. 3, pp. 181-185, 2010.

[18] B. F. El-Rayes, M. M. Zalupski, S. G. Manza et al., "Phase-II study of dose attenuated schedule of irinotecan, capecitabine, and celecoxib in advanced colorectal cancer," Cancer Chemotherapy and Pharmacology, vol. 61, no. 2, pp. 283-289, 2008.

[19] M. Kochi, W. Ichikawa, E. Meguro et al., "Phase II study of FOLFOX4 with "wait and go" strategy as first-line treatment for metastatic colorectal cancer," Cancer Chemotherapy and Pharmacology, vol. 68, no. 5, pp. 1215-1222, 2011.

[20] C. Du, Z. Z. Liu, Z. Ding, F. Guo, D. Ma, and X. Xie, "Autologous cytokine-induced killer cells combined with chemotherapy in 
the treatment of advanced colorectal cancer: a randomized control study," The Chinese-German Journal of Clinical Oncology, vol. 12, no. 10, pp. 487-491, 2013.

[21] X. Zhou, G. Xie, S. Wang et al., "Potent and specific antitumor effect for colorectal cancer by CEA and Rb double regulated oncolytic adenovirus harboring ST13 gene," PLoS ONE, vol. 7, no. 10, Article ID e47566, 2012.

[22] A. L. Small-Howard and H. Harris, "Advantages of the AMDLELISA DR-70 (FDP) assay over carcinoembryonic antigen (CEA) for monitoring colorectal cancer patients," Journal of Immunoassay and Immunochemistry, vol. 31, no. 2, pp. 131-147, 2010.

[23] H.-F. Qin, L.-L. Qu, H. Liu, S.-S. Wang, and H.-J. Gao, “Serum CEA level change and its significance before and after Gefitinib therapy on patients with advanced non-small cell lung cancer," Asian Pacific Journal of Cancer Prevention, vol. 14, no. 7, pp. 4205-4208, 2013.

[24] R. Petrioli, A. Licchetta, G. Roviello et al., "CEA and CA19.9 as early predictors of progression in advanced/metastatic colorectal cancer patients receiving oxaliplatin-based chemotherapy and bevacizumab," Cancer Investigation, vol. 30, no. 1, pp. 6571, 2012.

[25] B. Su, H. Shi, and J. Wan, "Role of serum carcinoembryonic antigen in the detection of colorectal cancer before and after surgical resection," World Journal of Gastroenterology, vol. 18, no. 17, pp. 2121-2126, 2012.

[26] C. J. Verberne, C. H. Nijboer, G. H. de Bock, I. Grossmann, T. Wiggers, and K. Havenga, "Evaluation of the use of decisionsupport software in carcino-embryonic antigen (CEA)-based follow-up of patients with colorectal cancer," BMC Medical Informatics and Decision Making, vol. 12, no. 1, article 14, 2012.

[27] M. Li, J. Li, A. Zhao et al., "Comparison of carcinoembryonic antigen prognostic value in serum and tumour tissue of patients with colorectal cancer," Colorectal Disease, vol. 11, no. 3, pp. 276281, 2009.

[28] S. Ito, H. Nakanishi, Y. Kodera, Y. Mochizuki, M. Tatematsu, and Y. Yamamura, "Prospective validation of quantitative CEA mRNA detection in peritoneal washes in gastric carcinoma patients," British Journal of Cancer, vol. 93, no. 9, pp. 986-992, 2005.

[29] X. Wei, X. Zhai, W. Zhao, D. Yang, and X. Han, "Research on the biological activity and anti-tumor effect against lymphoma cells of DC-CIK cells," Chinese-German Journal of Clinical Oncology, vol. 7, no. 11, pp. 666-669, 2008.

[30] S. Koido, T. Ohkusa, S. Homma et al., "Immunotherapy for colorectal cancer," The World Journal of Gastroenterology, vol. 19, no. 46, pp. 8531-8542, 2013.

[31] B. Rao, M. Han, L. Wang et al., "Clinical outcomes of active specific immunotherapy in advanced colorectal cancer and suspected minimal residual colorectal cancer: a meta-analysis and system review," Journal of Translational Medicine, vol. 9, article 17, 2011. 


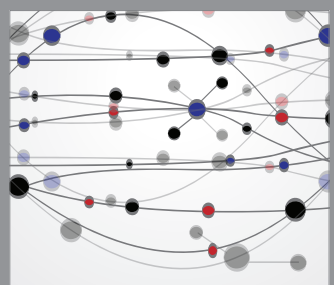

The Scientific World Journal
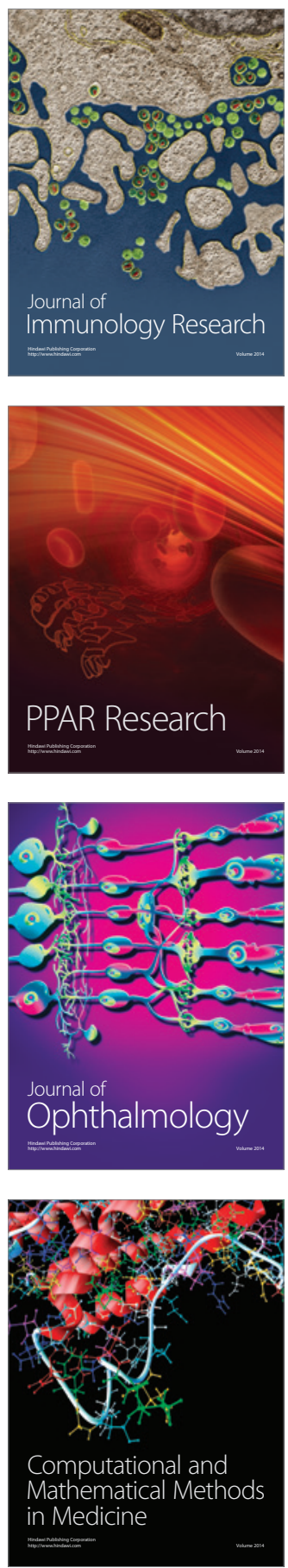

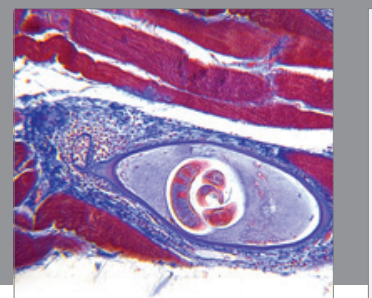

Gastroenterology

Research and Practice
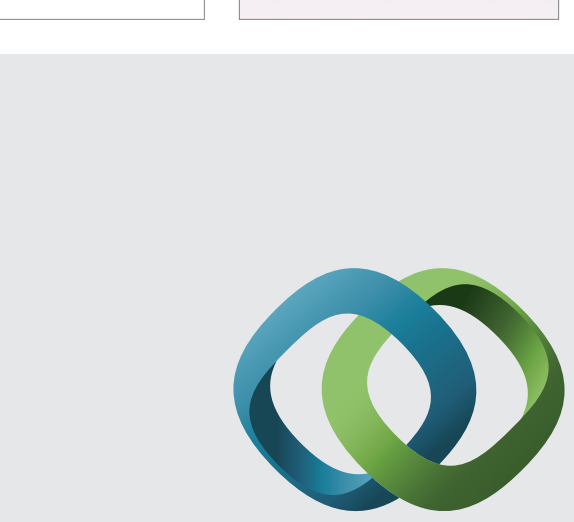

\section{Hindawi}

Submit your manuscripts at

http://www.hindawi.com
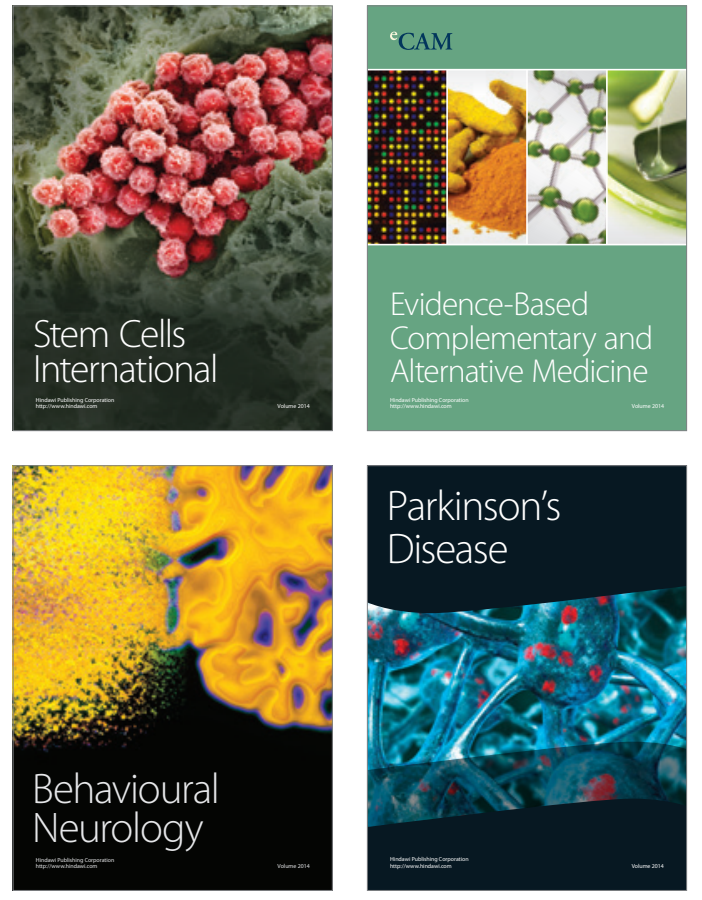
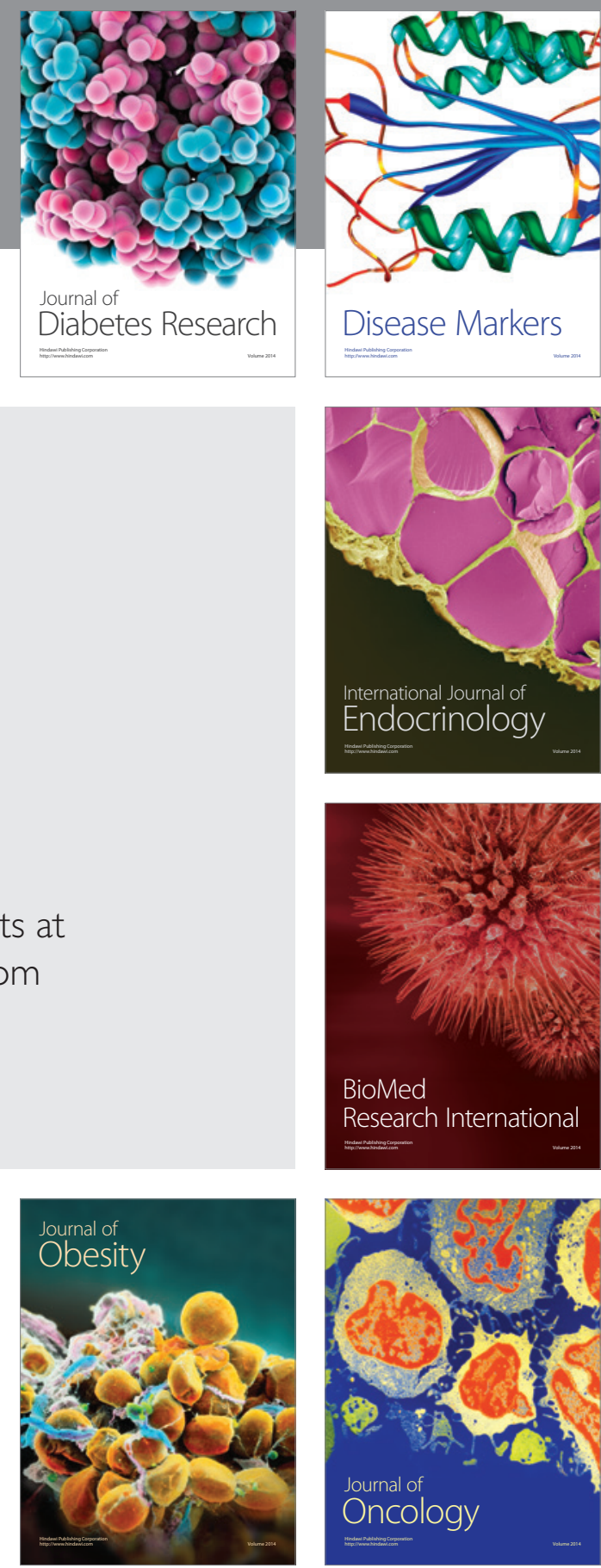

Disease Markers
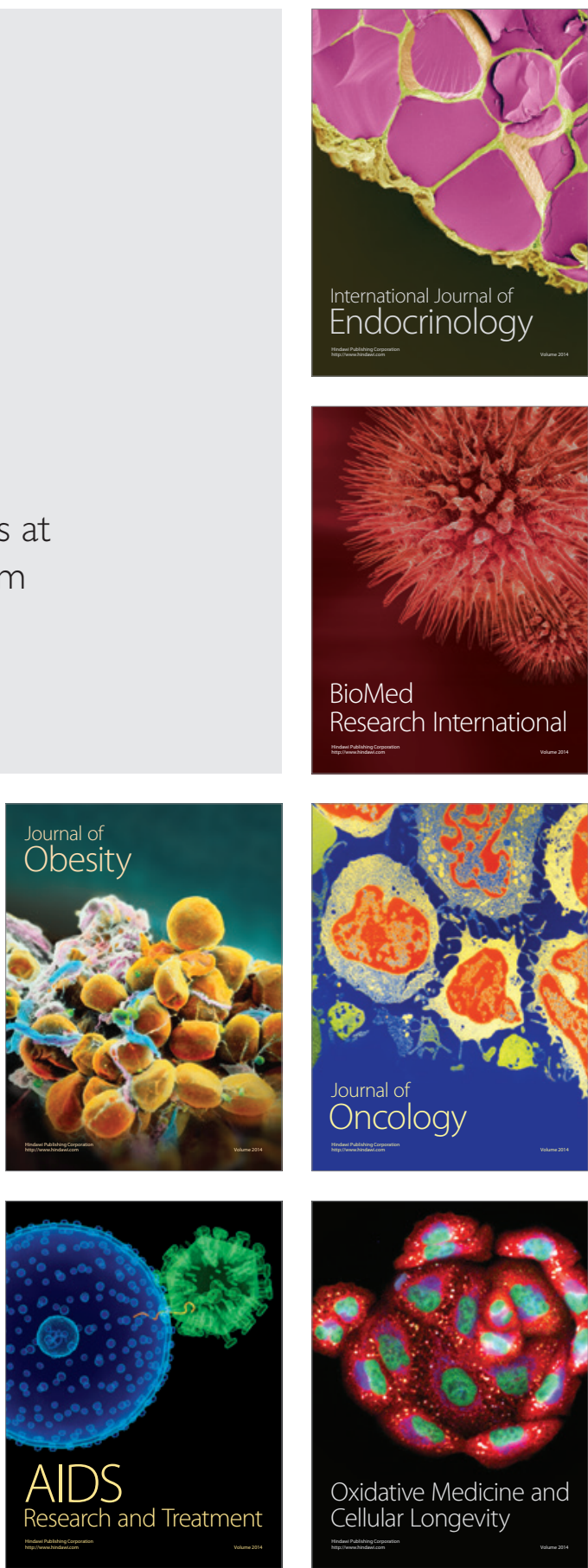